\title{
Clinical differentiation of psychogenic non- epileptic seizure: a practical diagnostic approach
}

\author{
I. Putu Eka Widyadharma ${ }^{1 *}$, Andreas Soejitno², D. P. G. Purwa Samatra ${ }^{1}$ and Anna M. G. Sinardja
}

\begin{abstract}
Background: Psychogenic non-epileptic seizure (PNES) has long been the counterpart of epileptic seizure (ES). Despite ample of evidence differentiating the two, PNES mistakenly diagnosed as ES was still common, resulting in unnecessary exposure to long-term antiepileptic medications and reduced patient's and caregiver's quality of life, not to mention the burgeoning financial costs.
\end{abstract}

Objectives: In this review, we aimed to elucidate various differences between PNES and epileptic seizure with respect to baseline characteristics, seizure semiology, EEG pattern, and other key hallmark features.

Methods: An unstructured search was carried out in PubMed, MEDLINE, and EMBASE using keywords pertinent to PNES and ES differentiation. Relevant information was subsequently summarized herein.

Results: PNES differs significantly with ES in terms of baseline characteristics, prodromal symptoms, seizure semiology, presence of pseudosleep, and other hallmark features (for instance provoking seizure with suggestion). The combined approach, if applied appropriately, can yield high diagnostic yield.

Conclusions: PNES can be clearly differentiated from ES via careful adherence to a set of valid clinical cues. The summarized clinical hallmarks is highly useful to prevent unnecessary ES diagnosis and treatment with AEDs.

Keywords: Psychogenic non-epileptic seizure, Epileptic seizure, Semiology

\section{Introduction}

Psychogenic non-epileptic seizures (PNES) is defined as paroxysmal changes of behavior, motor, sensory, autonomic, cognitive, and/or emotion which are not an epileptic seizure (ES) [1,2]. Despite its relatively simplistic definition, PNES continues to be a problem in which there is no clear-cut diagnostic criteria to differentiate it with ES. Moreover, the incidence of PNES is relatively high, accounting for approximately $5 \%$ of epilepsy incidence at 3-4 per 100,000 with prevalence of 2 to 33 per 100,000 persons $[3,4]$. In fact, it accounted for roughly $10-40 \%$ of patients with refractory seizures referred to

\footnotetext{
* Correspondence: eka.widyadharma@unud.ac.id

${ }^{1}$ Neurology Department, Faculty of Medicine Universitas Udayana/Sanglah Hospital, Denpasar, Bali 80114, Indonesia

Full list of author information is available at the end of the article
}

tertiary epilepsy centers [4-6]. Such numbers indicate that PNES is relatively common among patients with epilepsy. Given the inherent difficulty of distinguishing it with ES and the high prevalence of PNES among ES, a cavalier practice, particularly pertaining to estabilishing a diagnosis, may lead to high numbers of PNES patients being treated for epilepsy. Administration of unnecessary antiepileptic medications, particularly in the long-term as in the case of epilepsy teratment, poses them to serious adverse effects, not to mention the consequential financial burden of up to USD 4 billion [7]. Numerous studies had attempted to discern PNES from ES through various aspects. This include their basic demographic characteristics, risk factors, clinical and semiological profiles of the seizure, and other significantly related parameters. These efforts, supported by long-termvideo-EEG 
monitoring, had been successful in increasing the diagnostic sensitiviy and specificity of PNES. Herein, we would like to discuss the relevant features of PNES as opposed to ES from various aspects and how can this information be optimized, in conjunction with the videoEEG monitoring, to increase the rate of PNES detection.

\section{Methods}

This was a non-systematic literature review to discuss the clinical differentiation between PNES an ES. We performed an unstructured search in PubMed, MEDLINE, and EMBASE between February to April 2020 (with additional references included during the revision process of this manuscript) using keywords either stand alone or in combination related to clinical aspects and hallmarks of PNES and ES, including "PNES," "pseudoseizure," "epilepsy," "epileptic seizure," "ictal characteristics," "seizure differentiation," "motor phenomena," "non-motor phenomena," "seizure semiology," "prodromal symptoms," "post-ictal manifestation," "EEG," "video EEG," "risk factors," and "comorbidities." We did not apply any restrictions towards date and language of publication, publisher, types of manuscript (review article, case report/series, observational studies, randomized controlled trial (RCT), or systematic review), or manuscript status (pre-print, online first, full-text) except for the sole abstract. Relevance of references to be included in the study was decided manually.

\section{Results}

There were 59 relevant references included in this study, comprising 53 observational studies (comprising diagnostic tests, prospective and retrospective observational studies) and 6 unsystematic literature reviews. As many as 48 references discussed the clinical spectrum and characteristics of PNES compared to ES, 6 described PNES association with psychiatric disorders, 3 described patient's demographics, incidence, and prevalence, and 2 studies described cost analysis. Relevant information pertinent to the study objective was extracted and discussed as followed and summarised in Table 1.

\section{Demographics and baseline characteristics}

The majority of PNES patients had been consistently female, ranging from 50 to $87 \%$ [2, 16-19]. The reason for gender discrepancy towards PNES was unknown. However, it was presumed that personality disorders played a significant role in the development of PNES. Female patients with PNES was shown to have a significantly higher proportion of childhood abuse and anxiety [20]. PNES occurred at a later age when compared with frontal lobe seizure (FLS) [21].

The mean age of onset for PNES was 25.44 years, although late onset cases involving people with 60 years of age or above also occurred, albeit to a smaller proportion $[8,17]$.

\section{Video-EEG characteristics Prodromal symptoms}

Prodromal symptoms in PNES varied and were reported in $39 \%$ of patients. Although it is generally difficult to distinguish an aura preceding a seizure episode with prodromal phenomena of PNES, some clinical caveats do exist. The most frequent manifestations were behavioral, cognitive, and emotional changes [12]. In fact, more than half of PNES patients experienced dizziness, visual, auditory, or other complex sensations [9]. In addition, another study found that among 22 patients with PNES reportedly to have a preceding prodrome, two reported olfactory hallucination by having smelled a flower fragrance, one reported a micropsia, and the other eighteen reported non-specific symptoms, including funny feeling, dizziness, and non-lateralized numbness [18]. Meanwhile, the most commonly reported auras among ES patients were of motor symptoms such as forced head or eye deviation to one particular side (75\%), bilateral shaking and/or body stiffness (45\%), receptive or expressive aphasia (90\%), and aberrant emotional states (for instance fear, panic, anxiety) [59\%] [22]. There were only 23, 28, and $26 \%$ of ES patients reported auditory, somatosensory, and visual symptoms, respectively. Moreover, none complained of having an olfactory hallucination.

The general consensus of ES with aura is mostly perceived to be due to focal aware seizure with or without secondary generalization (primarily tonic clonic seizure) [23]. In contrast, it was reported that more than one third of PNES patients exhibited prodromal phenomena prior to atonic, absence, and persistent tonic behaviors, which if it had been a seizure, would have otherwise been highly unorthodox to be associated with an aura [18].

The onset of prodromes were also different between PNES and ES. The prodromal phenomena can either occur at the beginning or in the middle of the motor phenomena among PNES, while an aura will exclusively precedes ES [1]. Furthermore, PNES patients tended to have a more prolonged and wider duration of prodromal interval (ranging from hours to days). Prodromal symptoms with EEG alterations can also be found in patients with nonconvulsive status epilepticus [24].

\section{Onset}

PNES usually had a gradual onset, in contrast to ES which usually began abruptly. However, this was not always the case. Since some PNES were reported to have an abrupt onset as well [25].

PNES usually occurred during wakefulness, but there were also cases of PNES which occurred during apparent sleep [26]. The gradual onset was also accompanied by 
Table 1 Summary of "yellow" clinical characteristics (yellow flag checklist) of PNES patients

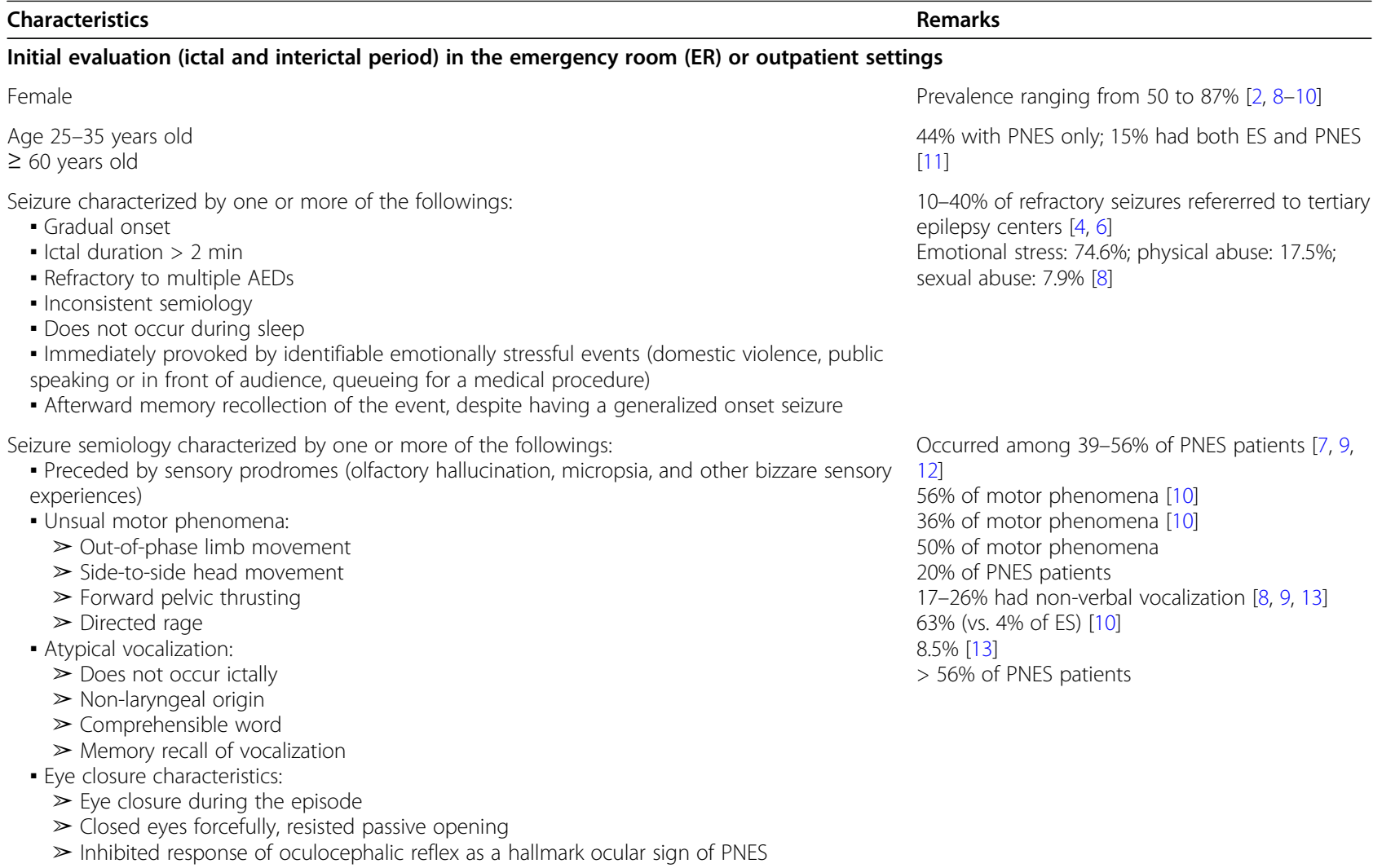

Post seizure motor manifestations comprising one or more of the followings:

Occured among $41 \%$ of PNES patients

- Retention of some movements (avoidance of falling arm, resistance to passive eye

movements, occasional eye blink, positive Henry and Woodruff sign, convergent gaze and eye

fixation upon mirror test)

- Absence of tongue-biting, urinary and/or fecal incontinence, fall-related trauma, or accidental

burn after an episode

- Normal corneal reflexes after an episode

- Absence of bilateral extensor plantar reflexes

- Rapid, shallow, quiet, irregular breathing pattern (resembling an exercise)

- Increased sympathetic activity before seizure and parasympathetic activity after seizure

- Weeping after event

Laboratory parameters:

- Absence of metabolic acidosis on blood gas analysis

- Serum prolactin levels $\geq 500 \mathrm{IU} / \mathrm{mL}$

Found in $28.8 \%$ of PNES patients and $84.4 \%$ of GTCS or partial seizure [14]

\section{Inpatient setting}

Association with psychiatric disorders

Tend to have cluster A or B personality disorder (primarily boderline personality disorder)

- Factitious disorder associated with litigation

- Previous history of sexual and physical abuse (including domestic violence)

Abbreviations: AED Anti-epileptic drugs, CI Confidence interval, ES Epileptic seizure, ER Emergency room, FLS Frontal lobe seizure, PNES Psychogenic non-epileptic seizure, TLS Temporal lobe seizure

fluctuating motor phenomena throughout the period (discussed later).

In all subjects, posterior alpha rhythm was present before the onset of seizure with no associated ictal or postictal EEG changes [26]. This were subsequently termed as pseudosleep by some authors $[1,27]$. Pseudosleep occurred exclusively in PNES, and none were observed among ES patients, yielding a sensitivity and specificity of 56 and 100\%, respectively [1]. In fact, this correlated with the finding that some PNES patients were able to recall what was happening during the period of acknowledged unresposiveness [28].

\section{Ictal duration}

PNES tended to have a longer and wider range of ictal duration than those of ES. The mean ictal duration of PNES was 148.7 s (95\% CI 115.2-191.8), significantly longer than that of ES patients $(47.7$ s; $95 \%$ CI $37.6-$ 
60.6) [29]. In fact, PNES usually had an ictal episode of longer than $2 \mathrm{~min}$, as opposed to those with generalized tonic clonic seizure (GTCS) and FLS which usually lasted less than $2 \mathrm{~min}$.

\section{Motor phenomena}

Motor phenomena were predominant in PNES, constantly reported in more than half of the patients in many studies. Out-of-phase limb movement (clonic) was prevalent, and it refers to an asynchronous jerk between left and right extremities and/or the movements being in an opposite direction between one and another [10]. In one study, it accounted for $56 \%$ of motor phenomenon for both upper and lower extremities movements, whereas none was observed in the control group [10]. In contrast, in-phase clonic movements, that is rhythmic jerking of both sides of upper and/or lower extremities, were observed significantly higher among those with ES than that of PNES patients (upper and lower extremity of 96 and $88 \%$ vs. 20 and 16\%, respectively). Another study found that motor phenomena comprised $81 \%$ of ictal manifestation among PNES patients, in which bilateral and unilateral motor phenomena were observed in 68 and 13\% of the patients, respectively [9]. Interestingly, $90 \%$ of the patients with multiple episodes had a stereotypical pattern which make it difficult to distinguish it with ES. In fact, one study categorized the stereotypical PNES semiology into six categories, comprising rhythmic motor, hypermotor, complex motor, dialeptic, nonepileptic auras, and mixed PNES [19].

Side-to-side head movement was observed in $36 \%$ of PNES as opposed to $8 \%$ in ES [10]. However, side-toside head movement was significantly more common in FLS than among any other types of seizure, comprising 11 seizures among 4 out of 11 patients with frontal lobe seizure (36\%) vs. 6 motor phenomena among 4 of 12 patients (33\%) with PNES [21]. Indeed, FLS is often mistaken as PNES, due to its confusing clinical manifestation. The difference may lie in the quality of the movement, in which PNES tended to have a violent and high amplitude movement, whereas ES demonstrated a less aggressive movement with slower frequency [10].

Pelvic thrusting was common among PNES patients. However, it was also prevalent in ES. Geyer and colleagues [30] demonstrated that pelvic thrusting can be seen in 24 and $12 \%$ of patients with FLS and temporal lobe seizure (TLS), respetively, while the same study also found pelvic thrusting in 17\% of PNES patients. Another study also found a comparable proportion of pelvic thrusting in FLS and PNES patients (55 vs. 50\%, respectively) [21]. Again, a discernable difference may lie in the quality of the thrust. As many as 44\% PNES patients showed forward thrust, while $12 \%$ of GTCS patients had backward thrust [10]. Although the backward thrust was also reported among PNES cases, none of GTCS patients had forward thrust [10, 31]. The forward pelvic thrust among PNES was also described as having a higher amplitude [30].

Other motor manifestations such as kicking, thrashing, and punching were less common. These are called as directed rage and belong to the hypermotor PNES on Seneviratne and colleagues.'s classification and composed of only $3.3 \%$ of all PNES. All of these violent movements were invariably bilateral and unprecedented $[18,19,32]$. Furthermore, semipurposeful movements were documented in several studies. Movements resembling epileptic automatisms were found in $20 \%$ of PNES [9]. In contrast, automatism was observed in ES and ES with PNES cases, but not in stand-alone PNES [33]. In PNES, however, the movement was described as more erratic and violent, with a tendency of goal direction $[1,9]$.

\section{Vocalization}

Vocalization during ictal episode was also common in both PNES and ES cases. Seventeen pecent to $26 \%$ of PNES cases had non-verbal vocalization as their clinical manifestation $[8,9,13]$. Meanwhile, ictal vocalization was highly prevalent among mixed and complex motor PNES (64.7 and 57.6\%, respectively) [19]. Vocalization in ES can be described as an ictal cry and tended to appear in the middle, rather than the beginning of the seizure [1]. In one study, the cry was described as of originating from the larynx (hence termed laryngeal sound) which was highly sensitive and specific $(85 \%$ and $100 \%$, respectively) for GTCS [34]. On the other hand, none of the vocalization in PNES occurred during seizure [10], and the manifestations varied, including moaning, coughing, with screaming or yelling being one of the commonest findings, and none expressed laryngeal sound as those observed in GTCS cases [12, 18, 34].

In addition, verbal responsiveness was found in a small proportion of PNES [1]. In fact, some patients with PNES had an understandable utterance [10]. While this can also be a perplexive sign to distinguish it with complex partial seizure (CPS), patients with PNES significantly had better memory recall than that of CPS patients (63\% vs. $4 \%$, respectively) [35]. Ictal stuttering was also identified among PNES cases, but none in ES. Although uncommon (reported in $8.5 \%$ patients with PNES), its presence was highly specific [36].

\section{Eye condition}

The majority of patients with PNES (more than 56\%) closed their eyes during seizure episode. A study employed video-recorded eye closure reported 92\% specificity of eye closure for PNES cases [37]. In another study, eye closure had a higher sensitivity and specificity for 
PNES (96.2 and 98.1\%, respectively) [38]. On the other hand, most ES patients (92-100\%) had their eyes opened during the beginning or in the middle of the episode [1, 38-40]. PNES patients also tended to forcefully close their eyes and resisted passive opening, whereas in ES patients, the eye closure was non-forceful [1]. In addition, one author had suggested the use of oculocephalic reflex to differentiate between PNES and other conditions [41]. Inhibited response accompanied by random saccadic eye movements may point the diagnosis more toward PNES than any organic neurological diseases.

\section{Post seizure manifestations}

Both patients with PNES and CPS had an impaired of consciousness after an episode. However, one study reported that as many as $41 \%$ of those with PNES with total unresponsiveness still retain some forms of conscious response, such as avoidance of falling arm or resistance to passive eye movements and $70 \%$ of these patients still had motor responses. This was in accordance with other studies which reported occasional eye blink, swallowing, or mouth movements post ictally [9].

One can also apply other non-invasive tests to prove that the patients still retain some or all of their consciousness after an episode, such as to drop patients' hand to their nasopharyngeal leads and to drop their heel on the contralateral shin and see if they would avoid hitting on it. Additional tests can be performed if the patients open their eyes after an episode, comprising when rolled on to the lateral side, the patients' gaze will deviate toward the ground and when rolled to the contralateral side, the gaze would still be fixated to the ground (so-called Henry and Woodruff sign), or when the patients were still in an episode, one can applied a small mirror in front of their faces and see if there is a convergent gaze and eye fixation. A termination of the motor phenomena can also be seen by using this technique [28]. Avoidance test was particularly helpful during an episode that mimicked absence or atonic seizure, as well as during post ictal episode of GTCS.

The natural behavior of PNES patients to protect themselves from harms and injuries may as well be reflected by the absence of any tongue-bite, urinary and/ or fecal incontinence, fall-related trauma, or accidental burn after an episode. In addition, all patients with GTCS reported post ictal confusion as opposed to $13 \%$ of PNES [39]. Other post ictal symptoms, including headache and fatigue were also less commonly reported in PNES cases, and the absence of such strongly indicate PNES [12, 42].

A significant proportion of PNES patients demonstrated a rapid (mean respiratory rate: PNES vs. GTCS 45.1 vs. $30.1 \mathrm{~s}, p<0.0001$, respectively), shallow (PNES vs. GTCS $87 \%$ vs. $0 \%, p<0.0001$, respectively), quiet
(PNES vs. GTCS $79 \%$ vs. $7 \%, p<0.0001$, respectively, and irregular breathing pattern (PNES vs. GTCS 79\% vs. $4 \%$, respectively) as opposed to that of ES patients comprising deep, regular, and loud (usually accompanied with snoring) [1, 12, 39]. Breathing pattern in PNES had a short inspiratory and expiratory phases, resembling an exercise. In addition, altered breathing pattern also lasted shorter than that of GTCS (94 vs. 357 s) [39].

There were also different patterns of autonomic nervous system (ANS) activation among patients with PNES and ES, with the latter had a greater activation. ES patients demonstrated a higher heart rate variability post ictally, indicating high sympathetic activity and reduced vagal tone [43]. Another study also found that heart rate increased significantly among PNES at $5 \mathrm{~min}$ before the seizure, while standard deviation of average beat-to-beat intervals and heart rate in low frequency domain decreased. The opposite findings were observed $5 \mathrm{~min}$ after the seizure subsided. This finding indicated an anticipated increased sympathetic followed by parasympathetic functionings during and after PNES, reflecting an increased arousal [44]. The relationship with emotion has yet to be studied. Thus, the heart rate variability can be used as another measure to differentiate between PNES and ES [45].

From an objective standpoint, ES patients usually presented with a sluggish corneal reflex or demonstrating bilateral extensor plantar reflexes (Babinski reflex) post ictally. These phenomena, particularly occur with prolonged seizure duration (including status epilepticus) by which it induced brain hypoxia and later on, brain edema. The prolonged hypoxic state can also be reflected in the systemic circulation by which a metabolic acidosis due to excessive lactate production by the contracting muscles can be observed on blood gas analysis [46].

Another useful biomarker to differentiate between PNES and ES would be to measure serum prolactin levels post ictally (the blood should be withdrawn between 10 and $20 \mathrm{~min}$ after a seizure). Serum prolactin levels rise to more than $500 \mathrm{IU} / \mathrm{mL}$ among $90 \%$ patients with GTCS and $60 \%$ patients with focal onset impaired awareness seizure, sparing normal values among focal onset aware seizure or a prolonged status epilepticus. Serum prolactin levels can sufficiently assist in differentiating GTCS from PNES (sensitivity 100\%, specificity 73\%). The result, however, should be interpreted with caution since prolactin levels were also elevated in PNES and syncope [14, 47]. A recent systematic review had documented that capillary prolactin levels had a sensitivity of $67.3 \%, 33.9 \%$, and $11.1 \%$ in diagnosing GTCS, focal impaired awareness seizures (FIAS), and focal aware seizures (FAS), respectively, with a very high median specificity of $99.1 \%$, suggesting that it could be quite useful in differentiating GTCS with PNES, but of limited 
usefulness in distinguishing between FIAS or FAS with PNES [48].

\section{Provoking seizure with suggestion}

In PNES, seizure can be induced by various strategies, including compression of the temple region, verbal suggestion, by applying tuning fork, moist swab, and torch, to saline injection [49]. The patient should receive a suggestion that these maneuvers could provoke a seizure prior to the test. All of these tests had been reported to have a $100 \%$ specificity for PNES diagnosis, with compression of the temporal region had the highest diagnostic yield (65.7\%). Another study involving patients with refractory epilepsy induced by intravenous saline injection also achieved $100 \%$ specificity among $91 \%$ of patients with PNES, as opposed to none in ES [50].

One can also use a more ethical approach, that is to modify the already performed routine procedure of EEG, such as applying photic stimulation and hyperventilation with accompanied suggestion to the patient that the purpose of this test was to provoke the seizure [51]. As many as $66 \%$ of PNES patients who received the stimuli with additional suggestion experienced an episode as opposed to only $33 \%$ who received identical stimuli but without an suggestion (Table 2).

\section{Association with psychiatric disorders}

Patients with PNES had been commonly associated with several psychiatric disorders. One study reported that as many as $47 \%$ of patients with PNES had some forms of psychiatric disorders and the other reported $100 \%$ prevalence of psychiatric diagnosis among PNES patients [2, 16]. Patients with PNES tended to have cluster A or B of personality type disorder, as opposed to cluster C in ES patients $[2,15]$. Furthermore, the authors had pointed that cluster $\mathrm{A}$ and $\mathrm{B}$ were considered highly dysfunctional likely to become the underlying pathology of PNES [52]. Another study found no significant difference in the proportion of depression, anxiety, post traumatic stress disorder, and malingering between patients with PNES and control [53]. However, PNES patients with ictal motor phenomena had a history of sexual and physical abuse. Similarly, Lancman and colleagues [54]. reported that history of sexual abuse was found in $10 \%$ of cases, andhysteroid traits, coping mechanism, and depression to be the most common underlying factors of PNES. Several studies had investigated the possibility of using MMPI-2 as an adjunct to video-EEG to diagnose PNES with relatively good yields (sensitivity and specificity ranging from 70 to $80 \%$ and $73-80 \%$, respectively) $[55,56]$.

\section{Discussion}

PNES, if diagnosed properly, is highly prevalent among patients with epilepsy with an estimated prevalence of 2 to 33 per 100,000 general population. Undiagnosed PNES lead to inappropriate patient treatment with unnecessary chronic administration of AEDs. Oftentimes, PNES patients underwent multidrug treatments because their seizure were apparently refractory to current medications. In fact, roughly one in five patients with apparent intractable seizure turned out to be having a nonepileptic seizure. This condition, consequently, predisposes patients to unnecessary harmful effects of AEDs, not to mention the significant financial burden resulting from a prolonged and burdensome diagnostic investigation, hospitalization, and ICU stay, as well as outpatient and emergency room visits [57]. The main problem of PNES lies in the diagnosis and its separation from ES. PNES often has multiple overlapping clinical features with that of ES to a certain extent that confidently differentiating between the two may be proven to be a daunting task. However, this does not mean that discerning them is impossible. PNES and ES possess many distinguishing clinical caveats as presented in this study. In fact, the ILAE Nonepileptic Seizures Task Force had already attempted to concept a staged approach to distinguish the two, emphasizing primarily according to patient's clinical history and physical examination, instead of vEEG [58]. The latter was considered a luxury that not every center may have the facility. Patients with

Table 2 EEG characteristics of PNES patients

\begin{tabular}{ll}
\hline Characteristics & Remarks \\
\hline EEG confirmation comprising one or more of the followings: & Occurred among 39-56\% of PNES patients [7, 9, \\
- Prodromal symptoms without EEG changes, sensory predominance (olfactory hallucination, & $12]$ \\
micropsia, other bizzare phenomena) & Successful inducetion in 65 to $84 \%$ of PNES (64) \\
- Pseudosleep & Successful induction of $65.7 \%$ and 0\% of PNES and \\
- Provoking seizure with suggestion: & ES, respectively [49] \\
$>$ Standard provocation (photic stimulation and hyperventilation) & Successful induction of $61.4 \%$ and 0\% of PNES and \\
$>$ Compression of the temple region & ES, respectively [49] \\
$>$ Applying tuning fork & Successful induction of 55.6\% and 0\% of PNES and \\
$>$ Intravenous saline injection & ES, respectively [49] \\
$>$ Verbal suggestion & Successful induction of 54.3\% and 0\% of PNES and \\
$>$ Torch light stimulation & ES, respectively [49] \\
& Successful induction of 40.7\% and 0\% of PNES and \\
& ES, respectively [49] \\
\hline
\end{tabular}


suspected PNES should be thoroughly screened with emphasis onconsideration of patients' demographics, detailed history taking, neuropsychological evaluation, and deliberate observation to delineate the semiology of the seizure along with its associated symptoms.

Clinicians are supposed to approach a case of seizure in a deliberate and systematic fashion. As an initial screening, there are several clinical "yellow flags" which raises the suspicion towards PNES, including atypical seizure semiology (discussed later), seizure that lasts more than 2 min, did not occur during sleep or if apparently sleeping can be demonstrated as a pseudosleep (confirmation with EEG later on), tends to occur among female, with onset of the first episode after the age of 10 in the absence of any secondary causes or risk factors of seizure, and previous history of any psychiatric disorders (particularly cluster A and B personality disorders [esp. borderline personality disorder], history of sexual abuse, and/or traumatic childhood experience) Table 1. An atypical seizure characterictics that could be categorized as "yellow flags" were as follows: preceded by predominantly sensory prodromes (olfactory hallucination, micropsia, and other bizzare sensory experiences), gradual onset, unsual motor phenomena (out-of-phase limb movement, side-to-side head movement, forward pelvic thrusting, and directed rage), atypical vocalization (does not occur ictally, non-laryngeal origin, comprehensible words, memory recollection of the event).

When most of these "yellow flags" appear, one would ideally proceed with EEG monitoring. Long-termvideoEEG monitoring (LTM) is regarded as the gold standard $[12,59]$. However, given that video-EEG monitoring is time- and resource-consuming, it should only be conducted on difficult cases wherein clinical evaluation with routine EEG failed to confirm the diagnosis.

We therefore proposed that patients with yellow flags should first undergo a standard EEG monitoring. During this period, an examiner can provoke a seizure by suggestion. If an episode turns out to occur, one should reevaluate the yellow flags checklist and see whether or not it match the previous descriptions. Any discrepancies should be documented and reviewed. EEG recordings should also be evaluated if it has any yellow flags. For instance, the patient was in a pseudosleep state (reflected by posterior awake alpha rhythm) either during prodromal symptoms or post episode. EEG recording is particularly helpful if one is still in doubt whether the patient has an FLS or PNES, in that FLS commonly arise during sleeping, tend to be brief (less than $30 \mathrm{~s}$ ) and ictal EEG can be observed during this period.

If a standard recording does not show any episodes or abnormalities, one can directly proceed to a 2 -h video EEG observation bearing the same goals and objectives, with yellow flag checklist in hand. During this state, it is rational to consider giving suggestion to a patient highly suspected with PNES. If a 2-h video EEG recording is still not confirmatory to provoke the occurrence of any seizure, one can proceed to a longer 4-h video EEG procedure. It is usually done consecutively as a continuation of the 2-h video EEG procedure.

One study reported that with extensive pre-LTMworkup, diagnostic uncertainty of PNES cases can be reduced to only $7 \%$ of patients [60]. Although LTM is considered as the gold standard for PNES diagnosis, some patients who underwent LTM were still having unclear diagnosis. In fact, about one in four patients had a non-diagnostic LTM [61]. Alving and colleagues [60] found that as many as $42 \%$ of recordings either had uncertain significant events $(25 \%)$ or no clinical events during LTM (17\%). The existing uncertain or absence of any clinical events can at least partially be backed by evidence gathered from periodic yellow flags checklist information. The corresponding information may act as a "blackbox" that record patient's activities and any significant events that appear as described in patient's history, during patient stay in the hospital ward, and during video EEG monitoring. Any discrepancies that appear in the process can further increase the index of suspicion for a PNES diagnosis.

Given the imperfect gold standard, the cost effectiveness of LTM should then be revisited, at least by comparing its diagnostic effectiveness with shorter duration of video-EEG monitoring. Indeed, one study found that $66 \%$ of PNES diagnosis can be confirmed with 1- to 2hvideo-EEG monitoring, while the remainder $34 \%$ were inconclusive, a number comparable to those of LTM [62]. However, 4-h video EEG had a significantly better ability to record clinical events (50.3 vs. 6.2\%) and higher sensitivity for both PNES (61.3 vs. 15.4\%) and ES (69.3 vs. 49.3\%) when compared to 1-h video EEG [63].

It is also important to investigate any underlying psychiatric disorders among patients with PNES. In our opinion, the involvement of any psychiatric disorders is necessary to be addressed at the earliest possible, just as important as defining whether or not it is an epileptic seizure. The corresponding physician should take a look at the previous medical record and to consult a psychiatrist as a routine evaluation whenever multiple yellow flags appear on examination. In our experience, immediate interdisciplinary management of patients suspected with PNES diagnosis can potentially lead to a better patient's cooperation and voluntary acknowledgement that they do not have a seizure, followed by efforts to not "get relapse" again. This approach can dramatically cut short the lengthy and burdensome diagnostic investigation that would otherwise be performed. In addition, by an immediate consult with a psychiatrist, one can also 
better discern if a PNES patient has any underlying factitious disorder for secondary gain purpose, particularly those related to legal issues (avoidance of litigation and imprisonment, financial fraud such as falsified insurance claim, and others).

Evidence regarding the difference between PNES and ES contains many subtle, but if carefully delinated, can be proven useful clinical clues. All of this information should further be sorted and prioritized according to its predictive value. Parameters with the highest diagnostic power would then be integrated into a scoring system, which in turn tested against LTM for its sensitivity and specificity. This concept has been documented before with high satisfactory results. For instance, Gates and colleagues applied multivariate discriminant analyses involving upper and lower extremities out-of-phase movement, pelvic thrust, and rigidity to diagnose a PNES. The resulting combination resulted in $96 \%$ of diagnostic accuracy [10]. The starting point would be to use this yellow flag checklist in a prospective diagnostic study and the following validation thereafter.

In the future, a combined approach of clinical scoring system and 4-h video EEG monitoring, with or without seizure induction strategies, and routine collaborative treatment with a psychiatrist as the earliest point possible is expected to be non-inferior or perhaps exceed the diagnostic yield when compared with LTM alone. When this occurs, a set of diagnostic criteria for PNES could then be established.

\section{Conclusion}

PNES is highly prevalent, resembling ES on many levels and poses significant problems for physicians. PNES can be carefully differentiated from ES with respect to its demographics, prodromal symptoms, onset, ictal duration, semiology of the seizure and its associated symptoms, and associated psychiatric disorders. Efforts to integrate the current wealth of evidence and using a more structured and combined diagnostic approach should be done in order to increase the accuracy of PNES diagnosis.

\section{Abbreviations}

AED: Anti-epileptic drugs; ANS: Autonomic nervous system; Cl: Confidence interval; CPS: Complex partial seizure; EEG: Electroencephalogram; ES: Epileptic seizure; ER: Emergency room; FLS: Frontal lobe seizure; GTCS: Generalized tonic clonic seizure; ILAE: International League Against Epilepsy; LTM: Long-term video EEG monitoring; MMPI-2: Minnesota Multiphasic Personality Inventory-2; PNES: Psychogenic non-epileptic seizure; TLS: Temporal lobe seizure

\section{Acknowledgements}

Not applicable.

\section{Authors' contributions}

IPEW and AS found and sorted the references, drafted, proofread, and wrote the manuscript. DPGPS and AMGS advised on the content, wrote, and final proofread of the manuscript. The authors have read and approved the manuscript.
Funding

None.

Availability of data and materials

Not applicable.

Ethics approval and consent to participate

Not applicable.

\section{Consent for publication}

Not applicable.

\section{Competing interests}

Not applicable.

\section{Author details}

${ }^{1}$ Neurology Department, Faculty of Medicine Universitas Udayana/Sanglah Hospital, Denpasar, Bali 80114, Indonesia. ${ }^{2}$ Neurology Resident, Faculty of Medicine Universitas Udayana/Sanglah Hospital, Denpasar, Bali 80114, Indonesia.

Received: 26 October 2020 Accepted: 12 January 2021

Published online: 05 February 2021

\section{References}

1. Mostacci B, Bisulli F, Alvisi L, Licchetta L, Baruzzi A, Tinuper P. Ictal characteristics of psychogenic nonepileptic seizures: what we have learned from video/EEG recordings--a literature review. Epilepsy Behav. 2011;22(2): 144-53.

2. Turner K, Piazzini A, Chiesa V, Barbieri V, Vignoli A, Gardella E, et al. Patients with epilepsy and patients with psychogenic non-epileptic seizures: videoEEG, clinical and neuropsychological evaluation. Seizure. 2011;20(9):706-10.

3. Szaflarski JP, Ficker DM, Cahill WT, Privitera MD. Four-year incidence of psychogenic nonepileptic seizures in adults in hamilton county, $\mathrm{OH}$. Neurology. 2000;55(10):1561-3.

4. Benbadis SR, Allen HW. An estimate of the prevalence of psychogenic nonepileptic seizures. Seizure. 2000:9(4):280-1.

5. Martin R, Burneo JG, Prasad A, Powell T, Faught E, Knowlton R, et al. Frequency of epilepsy in patients with psychogenic seizures monitored by video-EEG. Neurology. 2003;61(12):1791-2.

6. Sigurdardottir KR, Olafsson E. Incidence of psychogenic seizures in adults: a population-based study in Iceland. Epilepsia. 1998;39(7):749-52.

7. Nowack WJ. Epilepsy: a costly misdiagnosis. Clin Electroencephalogr. 1997; 28(4):225-8.

8. Patidar Y, Gupta M, Khwaja GA, Chowdhury D, Batra A, Dasgupta A. Clinical profile of psychogenic non-epileptic seizures in adults: a study of 63 cases. Ann Indian Acad Neurol. 2013;16(2):157-62.

9. Gulick TA, Spinks IP, King DW. Pseudoseizures: ictal phenomena. Neurology. 1982;32(1):24-30.

10. Gates JR, Ramani V, Whalen S, Loewenson R. Ictal characteristics of pseudoseizures. Arch Neurol. 1985;42(12):1183-7.

11. Kellinghaus C, Loddenkemper T, Dinner DS, Lachhwani D, Luders HO. Nonepileptic seizures of the elderly. J Neurol. 2004;251(6):704-9.

12. Ali S, Jabeen S, Arain A, Wassef T, Ibrahim A. How to use your clinical judgment to screen for and diagnose psychogenic nonepileptic seizures without video electroencephalogram. Innov Clin Neurosci. 2011;8(1):36-42.

13. Boon PA, Williamson PD. The diagnosis of pseudoseizures. Clin Neurol Neurosurg. 1993;95(1):1-8.

14. Abubakr A, Wambacq I. Diagnostic value of serum prolactin levels in PNES in the epilepsy monitoring unit. Neurol Clin Pract. 2016;6(2):116-9.

15. D'Alessio L, Giagante B, Oddo S, Silva WW, Solís P, Consalvo D, et al. Psychiatric disorders in patients with psychogenic non-epileptic seizures, with and without comorbid epilepsy. Seizure. 2006;15(5):333-9.

16. Meierkord H, Will B, Fish D, Shorvon S. The clinical features and prognosis of pseudoseizures diagnosed using video-EEG telemetry. Neurology. 1991; 41(10):1643-6.

17. Behrouz R, Heriaud L, Benbadis SR. Late-onset psychogenic nonepileptic seizures. Epilepsy Behav. 2006;8(3):649-50.

18. Luther JS, McNamara JO, Carwile S, Miller P, Hope V. Pseudoepileptic seizures: methods and video analysis to aid diagnosis. Ann Neurol. 1982; 12(5):458-62. 
19. Seneviratne $U$, Reutens D, D'Souza W. Stereotypy of psychogenic nonepileptic seizures: insights from video-EEG monitoring. Epilepsia. 2010; 51(7):1159-68.

20. Gamze A, Nesim K, Aytekin A, Orhan D. Dissociation and childhood abuse history in epileptic and pseudoseizure patients. Epileptic Disorders. 2004; 6(3):187-92.

21. Saygi S, Katz A, Marks DA, Spencer SS. Frontal lobe partial seizures and psychogenic seizures: comparison of clinical and ictal characteristics. Neurology. 1992;42(7):1274-7.

22. Dugan P, Carlson C, Bluvstein J, Chong DJ, Friedman D, Kirsch HE, et al. Auras in generalized epilepsy. Neurology. 2014;83(16):1444-9.

23. Scaramelli A, Braga P, Avellanal A, Bogacz A, Camejo C, Rega I, et al. Prodromal symptoms in epileptic patients: clinical characterization of the pre-ictal phase. Seizure. 2009;18(4):246-50.

24. Alving J, Beniczky S. Epileptic prodromes: are they nonconvulsive status epilepticus? Seizure. 2013;22(7):522-7.

25. Russell A. The diagnosis and management of pseudoseizures or psychogenic non-epileptic events. Ann Indian Acad Neurol. 2006;9:60.

26. Thacker K, Devinsky O, Perrine K, Alper K, Luciano D. Nonepileptic seizures during apparent sleep. Ann Neurol. 1993;33(4):414-8.

27. Benbadis SR, Lancman ME, King LM, Swanson SJ. Preictal pseudosleep: a new finding in psychogenic seizures. Neurology. 1996;47(1):63-7.

28. Mellers JD. The approach to patients with "non-epileptic seizures". Postgrad Med J. 2005;81(958):498-504.

29. Seneviratne U, Minato E, Paul E. How reliable is ictal duration to differentiate psychogenic nonepileptic seizures from epileptic seizures? Epilepsy Behav. 2017;66:127-31

30. Geyer JD, Payne TA, Drury I. The value of pelvic thrusting in the diagnosis of seizures and pseudoseizures. Neurology. 2000:54(1):227-9.

31. Leis AA, Ross MA, Summers AK. Psychogenic seizures: ictal characteristics and diagnostic pitfalls. Neurology. 1992;42(1):95-9.

32. Kanner AM, Morris HH, Luders H, Dinner DS, Wyllie E, Medendorp SV, et al. Supplementary motor seizures mimicking pseudoseizures: some clinical differences. Neurology. 1990;40(9):1404-7.

33. Devinsky O, Sanchez-Villasenor F, Vazquez B, Kothari M, Alper K, Luciano D. Clinical profile of patients with epileptic and nonepileptic seizures. Neurology. 1996;46(6):1530-3.

34. Elzawahry H, Do CS, Lin K, Benbadis SR. The diagnostic utility of the ictal cry. Epilepsy Behav. 2010;18(3):306-7.

35. Bell WL, Park YD, Thompson EA, Radtke RA. Ictal cognitive assessment of partial seizures and pseudoseizures. Arch Neurol. 1998;55(11):1456-9.

36. Vossler DG, Haltiner AM, Schepp SK, Friel PA, Caylor LM, Morgan JD, et al. Ictal stuttering: a sign suggestive of psychogenic nonepileptic seizures. Neurology. 2004:63(3):516-9.

37. Syed TU, Arozullah AM, Suciu GP, Toub J, Kim H, Dougherty ML, et al. Do observer and self-reports of ictal eye closure predict psychogenic nonepileptic seizures? Epilepsia. 2008:49(5):898-904.

38. Chung SS, Gerber P, Kirlin KA. Ictal eye closure is a reliable indicator for psychogenic nonepileptic seizures. Neurology. 2006;66(11):1730-1.

39. Azar NJ, Tayah TF, Wang L, Song Y, Abou-Khalil BW. Postictal breathing pattern distinguishes epileptic from nonepileptic convulsive seizures. Epilepsia. 2008;49(1):132-7.

40. DeToledo JC, Ramsay RE. Patterns of involvement of facial muscles during epileptic and nonepileptic events: review of 654 events. Neurology. 1996; 47(3):621-5.

41. Bounds JA. Ictal eye closure is a reliable indicator for psychogenic nonepileptic seizures. Neurology. 2007;68(12):963.

42. Ettinger AB, Weisbrot DM, Nolan E, Devinsky O. Postictal symptoms help distinguish patients with epileptic seizures from those with non-epileptic seizures. Seizure. 1999;8(3):149-51

43. Ponnusamy A, Marques $J$, Reuber M. Comparison of heart rate variability parameters during complex partial seizures and psychogenic nonepileptic seizures. Epilepsia. 2012;53(8):1314-21.

44. van der Kruijs SJ, Vonck KE, Langereis GR, Feijs LM, Bodde NM, Lazeron RH et al. Autonomic nervous system functioning associated with psychogenic nonepileptic seizures: analysis of heart rate variability. Epilepsy Behav. 2016; 54:14-9.

45. Ponnusamy A, Marques JL, Reuber M. Heart rate variability measures as biomarkers in patients with psychogenic nonepileptic seizures: potentia and limitations. Epilepsy Behav. 2011;22(4):685-91.
46. Lipka K, Bulow HH. Lactic acidosis following convulsions. Acta Anaesthesiol Scand. 2003:47(5):616-8.

47. Fisher RS. Serum prolactin in seizure diagnosis: glass half-full or half-empty? Neurol Clin Pract. 2016:6(2):100-1.

48. Wang Y-Q, Wen Y, Wang M-M, Zhang Y-W, Fang Z-X. Prolactin levels as a criterion to differentiate between psychogenic non-epileptic seizures and epileptic seizures: a systematic review. Epilepsy Res. 2021;169:106508.

49. Goyal G, Kalita J, Misra UK. Utility of different seizure induction protocols in psychogenic nonepileptic seizures. Epilepsy Res. 2014;108(6):1120-7.

50. Slater JD, Brown MC, Jacobs W, Ramsay RE. Induction of pseudoseizures with intravenous saline placebo. Epilepsia. 1995:36(6):580-5.

51. McGonigal A, Oto M, Russell AJ, Greene J, Duncan R. Outpatient video EEG recording in the diagnosis of non-epileptic seizures: a randomised controlled trial of simple suggestion techniques. J Neurol Neurosurg Psychiatry. 2002:72(4):549-51.

52. Harden $\mathrm{CL}$, Jovine L, Burgut FT, Carey BT, Nikolov BG, Ferrando SJ. A comparison of personality disorder characteristics of patients with nonepileptic psychogenic pseudoseizures with those of patients with epilepsy. Epilepsy Behav. 2009;14(3):481-3.

53. Abubakr A, Kablinger A, Caldito G. Psychogenic seizures: clinical features and psychological analysis. Epilepsy Behav. 2003;4(3):241-5.

54. Lancman ME, Brotherton TA, Asconape JJ, Penry JK. Psychogenic seizures in adults: a longitudinal analysis. Seizure. 1993;2(4):281-6.

55. Cragar DE, Berry DT, Fakhoury TA, Cibula JE, Schmitt FA. A review of diagnostic techniques in the differential diagnosis of epileptic and nonepileptic seizures. Neuropsychol Rev. 2002;12(1):31-64.

56. Schramke CJ, Valeri A, Valeriano JP, Kelly KM. Using the Minnesota Multiphasic Inventory 2, EEGs, and clinical data to predict nonepileptic events. Epilepsy Behav. 2007;11(3):343-6.

57. Seneviratne U, Low ZM, Low ZX, Hehir A, Paramaswaran S, Foong M, et al. Medical health care utilization cost of patients presenting with psychogenic nonepileptic seizures. Epilepsia. 2019;60(2):349-57.

58. LaFrance WC Jr, Baker GA, Duncan R, Goldstein LH, Reuber M. Minimum requirements for the diagnosis of psychogenic nonepileptic seizures: a staged approach: a report from the International League Against Epilepsy Nonepileptic Seizures Task Force. Epilepsia. 2013;54(11):2005-18.

59. Gedzelman ER, LaRoche SM. Long-term video EEG monitoring for diagnosis of psychogenic nonepileptic seizures. Neuropsychiatr Dis Treat. 2014;10: 1979-86.

60. Alving J, Beniczky S. Diagnostic usefulness and duration of the inpatient long-term video-EEG monitoring: findings in patients extensively investigated before the monitoring. Seizure. 2009;18(7):470-3.

61. Popkirov S, Jungilligens J, Gronheit W, Wellmer J. Diagnosing psychogenic nonepileptic seizures: Video-EEG monitoring, suggestive seizure induction and diagnostic certainty. Epilepsy Behav. 2017;73:54-8.

62. Benbadis SR, Siegrist K, Tatum WO, Heriaud L, Anthony K. Short-term outpatient EEG video with induction in the diagnosis of psychogenic seizures. Neurology. 2004;63(9):1728-30.

63. Nguyen-Michel VH, Dinkelacker V, Solano O, Levy PP, Lambrecq V, Adam C, et al. $4 \mathrm{~h}$ versus $1 \mathrm{~h}$-nap-video-EEG monitoring in an Epileptology Unit. Clin Neurophysiol. 2016;127(9):3135-9.

\section{Publisher's Note}

Springer Nature remains neutral with regard to jurisdictional claims in published maps and institutional affiliations. 